\title{
Does reducing interns' work hours reduce the rate of medical errors?
}

Landrigan CP, Rothschild JM, Cronin JW, Kaushal R, Burdick E, Katz JT, et al. Effect of reducing interns' work hours on serious medical errors in intensive care units. N Engl J Med 2004;351: 1838-48.

Background: Although multiple studies have shown that sleep deprivation affects physicians' abilities to perform various tasks, the impact of sleep deprivation on patient care remains

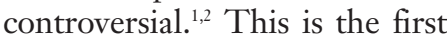
randomized trial to examine the impact of reduced physician work hours on medical error.

Design: This prospective, randomized single-centre intervention trial assigned interns to either a "traditional" on-call schedule every third night with extended work shifts of up to 28 hours or an "intervention" schedule that eliminated 24-hour shifts and decreased the number of scheduled hours per week. Rotations were of 4 weeks' duration in either the coronary care unit or the medical intensive care unit. The primary outcome was serious errors associated with interns. Trained physician observers were used as the primary method for detecting errors. Voluntary staff reporting, chart review and a computerized event-detection monitor were supplementary methods. All detected errors were verified and classified by blinded physician reviewers. Secondary end points included all serious errors, errors that were caught ("intercepted") by other staff and preventable adverse events.

Results: Interns worked about 79 hours per week during the traditional schedule and 63

hours per week during the inter-

vention schedule. The rate of serious medical errors made by interns was 36\% higher during the traditional schedule than during the intervention schedule (136 v. 100 per 1000 patientdays, $p<0.001)$. The rate of nonintercepted serious errors was also higher during the traditional schedule (44.8 v. 28.6 per 1000 patient-days, $p=0.02$ ), as was the rate of all serious errors (193.2 v. 158.4 per 100 patientdays, $p<0.001)$. No difference in the rates of preventable adverse events was detected (38.6 for the traditional schedule $v$. 38.5 for the intervention schedule per 1000 patient-days).

Commentary: An accompanying study suggested that the observed reduction in medical errors is explained by more hours of sleep and fewer attentional failures among interns on the intervention schedule. ${ }^{3}$ Although a blinded study physician reviewed all potential errors and events, a potential weakness of the study was the lack of blinding of the interns, staff and other observers. However, such blinding would be virtually impossible to achieve. The reported reduction in serious errors is intriguing, but the real goal is to avoid harm to patients. This study was not designed to detect an effect on preventable adverse events, so the impact of reduced work hours on patient safety remains to be defined. It is also important to note that the study group created a system for careful sign-over of patients to minimize errors associated with cross-coverage by multiple physicians.

How would this intervention play out in other settings? More experienced physicians may be less susceptible to serious errors when fatigued. Error rates would likely be lower outside of intensive care unit settings, so the impact of reduced work hours would be less. Moreover, many hospitals lack the sophisticated methods to detect errors that were used in this study, so reductions in error would not be immediately apparent in typical clinical settings.

Practice implications: The study results are not surprising to any clinician who has received signover from a fatigued colleague after a night on call. Still, the rigorous methods and promising results of this study should stimulate additional attention on physician fatigue and sleep deprivation. Residency training programs, particularly those with rotations in intensive and coronary care units, should note the uncomfortably high rate of nonintercepted serious errors. At a minimum, multicentre trials of reduced physician work hours should be undertaken in teaching and nonteaching hospitals. Further, physicians should document their shift lengths and total hours worked per week, so that the latent hazard of physician fatigue becomes more visible to hospitals and governments. The intervention in this study was accomplished by adding an extra intern to the call pool. Medical schools, hospitals and governments will need to plan for additional physician resources to achieve similar results.

Michael Bernstein

Division of General Internal

Medicine

Edward E. Etchells

Division of General Internal

Medicine, Patient Safety Service

Sunnybrook and Women's College

Health Sciences Centre

Toronto, Ont.

\section{References}

1. Weinger M, Anconi-Israel S. Sleep deprivation and clinical performance. 7AMA 2002;287:955-7.

2. Laine C, Goldman L, Soukup J, Hayes $\mathrm{J}$. The impact of restricting medical house staff working hours on the quality of patient care. FAMA 1993;269:374-8.

3. Lockley SW, Cronin JW, Evans EE, Cade BE, Lee CJ, Landrigan CP, et al. Effect of reducing interns' weekly work hours on sleep and attentional failures. NEngl f Med 2004;351:1829-37. 